\title{
Context Effects in the Discriminability of Spatial Cues
}

\author{
Julia Kerstin Maier, ${ }^{1,2,3,4}$ David McAlpine, ${ }^{3}$ Georg M. Klump, ${ }^{4}$ and Daniel Pressnitzer ${ }^{1,2}$ \\ ${ }^{1}$ CNRS UMR 8158, Université Paris Descartes, Paris, France \\ ${ }^{2}$ Département d'Etudes Cognitives, Ecole Normale Supérieure, 29 rue d'Ulm, 75230 Paris Cedex 05, France \\ ${ }^{3}$ UCL Ear Institute, University College London, 332-336 Gray's Inn Road, London, WC1X 8EE UK \\ ${ }^{4}$ Animal Physiology E Behavior Group, Institute for Biology and Environmental Sciences, Carl von Ossietzky University \\ Oldenburg, 26111 Oldenburg, Germany
}

Received: 29 June 2009; Accepted: 25 November 2009; Online publication: 22 December 2009

\begin{abstract}
In order to investigate whether performance in an auditory spatial discrimination task depends on the prevailing listening conditions, we tested the ability of human listeners to discriminate target sounds with and without presentation of a preceding sound. Target sounds were either lateralized by means of interaural time differences (ITDs) of $+400,0$, or $-400 \mu$ s or interaural level differences (ILDs) with the same subjective intracranial locations. The preceding sound was always lateralized by means of ITD. This allowed for testing whether the effects of a preceding sound were location- or cue-specific. Preceding sounds and target sounds were randomly paired across trials. Listeners had to discriminate whether they perceived the target sounds as coming from the same or different intracranial locations. Finally, stimuli were selected so that, without any preceding sound, ITD and ILD cues were equally discriminable at all target lateralizations. Stimuli were $800 \mathrm{~Hz}$-wide, 400-ms duration bands of noise centered at $500 \mathrm{~Hz}$, presented over headphones. The duration of the preceding sound was randomly selected from a uniform distribution spanning from 1s to 2s. Results show that discriminability of both binaural cues was improved for midline target positions when preceding sound and targets were co-located, whereas it was impaired when preceding sound and targets came from different positions. No effect of the preceding sound was found for left or right target positions. These results are compatible with a purely bottom-up mechanism based on adaptive coding of ITD around the midline that may be combined with top-down mechanisms to increase localization accuracy in realistic listening conditions.
\end{abstract}

Correspondence to: Julia Kerstin Maier · Département d'Etudes Cognitives · Ecole Normale Supérieure $\cdot 29$ rue d'Ulm, 75230 Paris Cedex 05, France. Telephone: +33-144-322673 email: jkmaier@ googlemail.com
Keywords: binaural adaptation, human, auditory, sound localization, psychoacoustics

\section{INTRODUCTION}

In realistic auditory scenes, sound localization is an ongoing process: some sound sources may be active for some period of time, but new sources may also suddenly appear in totally different locations. To understand fully spatial hearing in natural situations, it is, therefore, important to know how sound localization at one moment in time is affected by preceding sounds.

A number of psychophysical studies have investigated the influence of preceding sounds on localization of subsequent sounds. After prolonged exposure to a preceding sound at a fixed location, an auditory spatial aftereffect can be observed (Thurlow and Jack 1973; Kashino and Nishida 1998; Dong et al. 1999; Carlile et al. 2000; Phillips and Hall 2005; Vigneault-MacLean et al. 2007). In all of these studies, the apparent location of the test sound is shifted away from the preceding sound's position. The first question raised by these experiments is whether the aftereffect reflects a criterion shift at the decision stage or true sensory adaptation. Against the criterion interpretation, Kashino and Nishida (1998), Phillips and Hall (2005), and Vigneault-MacLean et al. (2007) showed that the aftereffect is frequency-specific: that is, preceding sound and test tone must share the same frequency region for the aftereffect to occur. The second question is whether the effect of the preceding sound is location- or cue-specific. Again using an apparent localization task, Phillips et al. (2006) showed that adaptation to interaural time difference (ITD) could transfer to interaural level difference (ILD), and vice versa, suggesting that adaptation is not cue-specific. 
Other studies have investigated whether the presence of a preceding tone influences localization performance for subsequent targets. Spence and Driver (1994) showed that a preceding sound located in a given hemifield reduced reaction times for localization tasks in the same hemifield. Getzmann (2004) showed that a preceding sound co-located with a target induced an improvement in azimuth discrimination performance. Importantly, both of these studies used free-field presentation, which combines effects of ITD and ILD. It is, therefore, possible that the effects observed are influenced by monaural adaptation to sound level. A similar paradigm was further investigated by Sach et al. (2000) using a measure of discriminability instead of reaction time and with cueing by ITD only. Results were inconclusive when the preceding sound was not indicative of the target location, with an improvement of performance in only two out of four subjects (their Experiment 1). Finally, Kashino (1998) reported a set of experiments where ITD discrimination was measured following long exposure to ITD only. An improvement in ITD discrimination performance was observed, both for midline and lateralized ITD. The effect was frequency-specific, suggesting a true sensory adaptation to ITD.

Sensory systems are known to change their responses to the prevailing sensory environment, which, we hypothesize, might contribute to the variety of behavioral data obtained after presenting a preceding sound. Neural responses to frequency (Ulanovsky et al. 2003), intensity (Dean et al. 2005), or ITD (Malone et al. 2002; Furukawa et al. 2005) have all been shown to depend on stimulus history. Recently, this has been termed "adaptive coding"; adaptive coding designates any type of change in the neural activity (not only a reduction in firing rate) that results in a more efficient encoding of the stimuli (e.g., see Dean et al. 2005 for evidence of adaptive coding of intensity in the auditory modality and Fairhall et al. 2001 for adaptive coding in the visual modality). For spatial hearing, Furukawa et al. (2005) showed that the presentation of short preceding tones with a given ITD could change the subsequent neural responses in the inferior colliculus (IC) in a way qualitatively consistent with the auditory localization aftereffect.

Here, we examined the effect of a preceding sound that contained a fixed ITD on subsequent ITD and ILD discrimination. Our approach addresses several issues that remain unresolved. First, single-neuron recordings suggest that neurons adapt almost fully to novel sound distributions in less than 1s (Dean et al. 2008). In contrast, the study of Kashino (1998) used prolonged adaptation (60s followed by 5 s top-ups). While Getzmann (2004) found a benefit for preceding sounds of 3s, it may be ascribed to ITD, ILD, or monaural adaptation. Here, psychophysical data were collected with time constants closer to those suggested from neurophysiological recordings (approx. 1s; Dean et al. 2008). Second, both Kashino (1998) and Getzmann (2004) used a single spatial location for the preceding sound in any given block, which may not be fully representative of the dynamic changes that arise in realistic auditory scenes. Finally, the interesting question raised by Phillips et al. (2006) concerning the transfer of adaptation to ITD or ILD cues has not yet been investigated with measures of discrimination performance.

To address these issues, we measured spatial discrimination performance after the presentation of a relatively short preceding noise burst (values were randomly selected from a uniform distribution spanning from 1s to 2s). We used signal detection theory (Green and Swets 1966, Macmillan and Creelman 2005) to estimate both changes in sensitivity $\left(d^{\prime}\right)$ and criterion $(c)$. The measures were performed for three different subjective intracranial locations (left, middle, right) that were randomly presented within the same experimental block. Finally, preceding sounds only contained an ITD cue, but discrimination was evaluated for both ITD and ILD targets. Importantly, discrimination performance without any preceding sound was first equated for all binaural cues and locations.

\section{METHODS}

\section{Subjects}

Seven subjects aged between 22 and 40 years participated in this study. All subjects had normal audiograms (i.e., better than $20 \mathrm{~dB}$ HL at frequencies between 0.125 and $8 \mathrm{kHz}$ in octave steps). Sensitivity differences between left and right ear thresholds at each frequency were smaller than $5 \mathrm{~dB}$ HL for all subjects.

\section{Stimuli and apparatus}

All stimuli were Gaussian noise bands filtered between 100 and $900 \mathrm{~Hz}$ (brickwall FFT filter). Target stimuli were $400 \mathrm{~ms}$ in duration, including $5 \mathrm{~ms}$ raised-cosine onset and offset ramps. The duration of preceding sounds was randomized between $1 \mathrm{~s}$ and $2 \mathrm{~s}$ (values were randomly selected from a uniform distribution spanning from $1 \mathrm{~s}$ to $2 \mathrm{~s}$, with a one-sample resolution), also including onset and offset ramps. The stimuli were lateralized with reference ITDs of $0 \mu$ s (middle), $+400 \mu \mathrm{s}$ (left), and $-400 \mu \mathrm{s}$ (right), as well as equivalent ILDs, measured as described below. Interstimulus intervals within the target sequence as well as between target sequence and preceding sound (where applicable) were $500 \mathrm{~ms}$, chosen to limit the influence of binaural sluggishness. Intertrial intervals were of 1.5 -s duration. 
Stimuli were computed online using Matlab on a personal computer and played back with a Creative SB Audigy 2ZS sound card (44.1 kHz sampling rate). They were then passed into a sound-attenuating booth (Industrial Acoustics type IAC 1202A) and presented to the subjects over headphones (Sennheiser HDA 200). Mean overall level was $68 \mathrm{~dB}$ SPL.

\section{Experimental procedures and data analysis}

The experiment consisted of three preliminary steps that were designed to equate subjective location and discriminability across binaural cues in the absence of a preceding sound, followed by the main discrimination task. In preliminary Step 1, ILDs were matched to ITDs $(0,+400,-400 \mu \mathrm{s})$ to produce identical subjective locations for each subject individually. In preliminary Step 2, discrimination thresholds were estimated with an adaptive procedure for both ITD and ILD cues at all intracranial locations. In preliminary Step 3, a constant stimulus task was used to find the physical differences in ITD and ILD required to obtain equal discriminability $\left(d^{\prime}=2\right)$ in the absence of a preceding sound, at all intracranial locations, for each subject individually. Finally, these values were used in the main task where the effect of the preceding sound on ITD and ILD discriminability was measured.

Preliminary Step 1: matching subjective locations of ITDs and ILDs

ITD references were fixed at $+400,0$, and $-400 \mu$ s (left, middle, right), all with an ILD of $0 \mathrm{~dB}$. ILD equivalents, required to produce the same apparent location as ITD references, were determined by employing a subjective matching paradigm. During any given trial, subjects could play one ITD reference by activating a button on a computer screen by mouse-click, with a free number of repeats. They could also play an ILD pointer (ITD always $0 \mu \mathrm{s}$, variable ILD) by activating a second button. Subjects could control the subjective location of the ILD pointer by using arrows to the left or the right, which shifted the ILD in steps of $2 \mathrm{~dB}$. The initial position of the ILD pointer was randomized from trial to trial, as was the ITD reference. Each subject took part in 15 practice trials per reference. Subsequently, the ILD equivalent for each subject was determined by averaging the ILD values derived from 30 trials per ITD reference.

\section{Preliminary Step 2: measuring just noticeable differences}

Following the reference matching task, just noticeable differences (JNDs) were measured for the three ITD and ILD values in a two-alternative-forced-choice paradigm. The task was to indicate which of two noise bursts deviated from a reference noise burst (XAB odd-one-out task). For references lateralized to the right or to the left, the deviation always occurred towards the midline. For references in the middle, the deviation was arbitrarily chosen to always occur to the left. Feedback was provided after each trial. An adaptive, 3-down-1-up method was used ( $d^{\prime}$ corresponding to 1.16). The difference in ITD in microseconds or ILD in decibels was adjusted by $50 \%$ for the first two reversals, $25 \%$ for the next two reversals, and $10 \%$ for the last four reversals. JNDs were taken as the average of the last four reversals. Each subject completed two practice sessions followed by five experimental sessions per reference. These seven sessions for a given reference (ITD or ILD, left, right, or middle) were completed consecutively. The order of the six references was varied randomly between subjects.

Preliminary Step 3: matching the ITD and ILD in the condition without preceding sound to a $d^{\prime}$ of 2

The third preliminary step was designed to find ITD and ILD increments that lead to performance of $d^{\prime}=2$ for each subject, binaural cue, and target location. The method of constant stimuli was used, with a samedifferent task. Subjects had to indicate if two consecutively presented noise bursts were presented from the same or different intracranial locations. In trials in which a difference was introduced, noise bursts were separated by one and three JNDs for ITDs or by one and two JNDs for ILDs. These differences were chosen after informal testing in order to try to bracket the value of $d^{\prime}=2$. Both spatial cues were presented randomly interleaved within one session. Further, the order of the reference and deviant intervals was randomly varied from trial to trial. Subjects completed one practice session followed by 12 experimental sessions comprising 96 trials each (1,152 trials per subject). Differences in ITD and ILD corresponding to a $d^{\prime}$ of 2 were then determined using linear interpolation. It is currently unknown whether the relationship between physical cues to ITD and ILD and discriminiability in terms of $d^{\prime}$ is linear or not. The present method was chosen only as a simple heuristic to find ITD and ILD increments leading to $d^{\prime}$ values of about 2 . Note that the validity of such a method is independently assessed in the main discrimination experiment: to the extent that the approach worked, $d^{\prime}$ should be equal to 2 in the "no preceding sound" condition for all subjects, all lateral angles, and both binaural cue types.

Main task: discriminating intracranial same-different locations with and without preceding sounds

In the main experiment, discriminability $\left(d^{\prime}\right)$ was measured for the ITD and ILD values determined in preliminary Step 3, with and without exposure to a preceding sound containing ITDs. Once more, subjects 
had to indicate whether or not two consecutively presented noise bursts were the same or different (Fig. 1). Subjects performed 25 sessions comprising 192 trials each $(4,800$ trials per subject). Because of the similarity to the task of Step 3, no practice session was conducted. The discriminability index $\left(d^{\prime}\right)$ and decision criterion $(c)$ were measured using signal detection theory with the following formulae:

$$
\begin{gathered}
d^{\prime}=z(\text { hits })-z(\text { false alarms }) \\
c=-0.5 \times(z(\text { hits })+z(\text { false alarms }))
\end{gathered}
$$

where " $z$ " denotes the number of standard deviations from the mean, "hits" the rate of detection of real differences in intracranial location, and "false alarms" the rate of identical stimuli incorrectly classified as different. Following the standard assumptions of signal detection theory, the above formulae posit a model in which the decision variables are Gaussian variables with equal variance (Macmillan and Creelman 2005). Each $d^{\prime}$ and $c$ value was computed over 200 repeats of trials.

\section{RESULTS}

Preliminary steps

Step 1. The subjective matching of location between fixed ITDs and variable ILDs is presented in

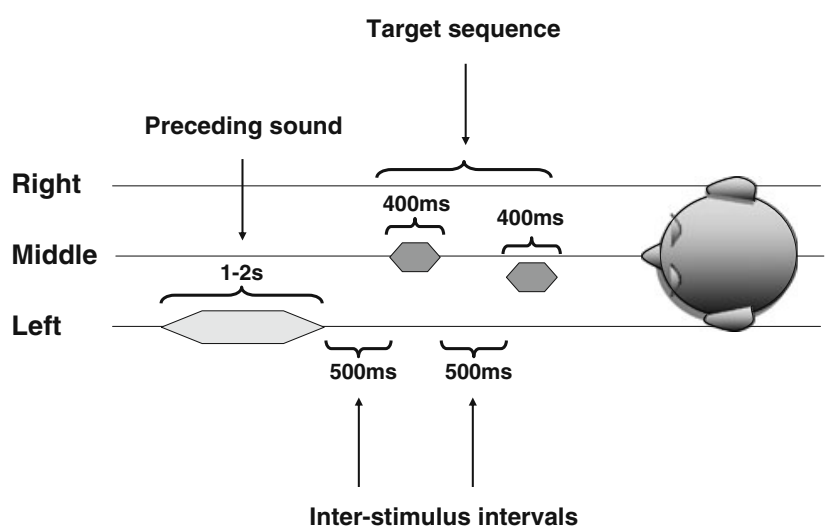

FIG. 1. Example of a trial of the main experiment. In this example, a preceding sound and target sound sequence are presented in different apparent locations. Subjects had to indicate whether the target sounds came from the same or different intracranial locations, which were presented equiprobably (here, the correct answer is "different"). Preceding sounds, when present, were always lateralized by means of ITDs. Target sounds were lateralized by means of ITDs in some trials or by ILDs matched to the ITD locations in other trials. The physical difference between targets was adjusted in order to produce a fixed level of discriminability when no preceding sound was present, for all binaural cues and locations. The location, presence/absence of the preceding sound, the location of the targets, and the type of target cue (ITD or ILD) were all randomly interleaved within an experimental block.
Table 1. Matches are highly consistent for the middle position (ITD $=0 \mu \mathrm{s})$. Greater variability is observed for lateralized ITDs, both within and across subjects.

Step 2. The JNDs for all cues and locations are shown in Table 2. The relatively large variability observed is consistent with published measurements (e.g., Mossop and Culling 1998).

Step 3. The number of JNDs estimated for equal discriminability of $d^{\prime}=2$ without preceding sounds is shown in Table 3, for all binaural cues, intracranial locations, and listeners. The same results are displayed again in terms of actual ITD and ILD values in Table 4 . From Table 3, it is apparent that large differences in terms of the estimated number of JNDs are required to attain equal discriminability for different cues (ILDs and ITDs) and also for different subjects. This is not what might have been predicted on the basis of the findings of Terhardt (1968), who suggested that the same number of JNDs should produce the same perceptual difference irrespective of the perceptual dimension. There are important differences between the two studies, however. Terhardt (1968) used a subjective measure on the dimensions of pitch, loudness, and roughness, whereas we used a discriminability measure on spatial cues.

Main task: how a preceding sound influences discriminability of binaural cues

Mean discriminability $\left(d^{\prime}\right)$ for each target location (left, middle, and right), binaural cue (ITD and ILD), and preceding sound condition (none, ITD left, ITD middle, and ITD right) are plotted in Figure 2A, with the corresponding individual data shown in Figure 2B. Without any preceding sound, discriminability is similar for both binaural cues and all target locations, indicating that the adjustment steps used to equate performance worked well on average (only the average result for ILD left is below the expected value). An effect of the preceding sound condition is observed for midline targets (Fig. 2A, middle panel). Here, discriminability tends to be better when preceding sound and target are co-located and poorer when preceding sound and target location are mismatched, on either side. Discriminability for the condition without preceding sound falls in between the central- and lateralpreceding-sound conditions. This pattern is not observed for the left and the right target locations (Fig. 2A, left and right panels, respectively).

A three-way repeated-measures ANOVA with factors of preceding sound condition, target location, and 
TABLE 1

Mean and standard deviation for ILD matches to reference ITDs for each subject and intracranial location (results of Step 1)

\begin{tabular}{|c|c|c|c|c|c|c|}
\hline \multirow{3}{*}{$\begin{array}{l}\text { Reference ITD [us] } \\
\text { Subject }\end{array}$} & \multicolumn{6}{|c|}{ Matched ILD [dB] } \\
\hline & \multicolumn{2}{|c|}{+400 (left) } & \multicolumn{2}{|c|}{$O$ (midline) } & \multicolumn{2}{|c|}{-400 (right) } \\
\hline & Mean & $S D$ & Mean & $S D$ & Mean & $S D$ \\
\hline 1 & 17.49 & 3.29 & 1.62 & 2.01 & -19.51 & 1.52 \\
\hline 2 & 16.13 & 1.91 & 0.42 & 1.02 & -11.15 & 1.68 \\
\hline 3 & 15.30 & 3.58 & 1.29 & 0.41 & -14.82 & 2.80 \\
\hline 4 & 7.19 & 2.03 & 0.65 & 1.69 & -5.44 & 1.17 \\
\hline 5 & 7.77 & 1.82 & -0.16 & 2.26 & -7.66 & 2.91 \\
\hline 6 & 11.77 & 3.96 & 0.06 & 1.41 & -14.98 & 4.40 \\
\hline 7 & 8.57 & 2.80 & -0.99 & 1.88 & -14.31 & 3.32 \\
\hline
\end{tabular}

binaural cue was performed. It revealed main differences in discriminability between preceding sound conditions $\left(P=0.015, F_{3,164}=4.567\right)$. In contrast, discriminability did not differ significantly between target locations $\left(P=0.812, F_{2,165}=0.211\right)$, nor between binaural cues $\left(P=0.915, F_{1,166}=0.012\right)$. The only significant interactions were found between preceding sound condition and target location $(P=0.001$, $\left.F_{6,161}=4.925\right)$ and between preceding sound condition and binaural cue $\left(P=0.014, F_{5,162}=4.623\right)$.

The main aim of the experiment was to evaluate the effect of a preceding sound on binaural discrimination performance. We expected that the effect of the preceding sound would be different if preceding sound and targets were co-located compared to when they were mismatched in intracranial location. In addition, we wanted to know whether the effect of a preceding sound lateralized by means of ITD could be observed for discriminability of an ILD target as well as an ITD target. To test for these effects, for each binaural cue, we carried out planned comparisons of discriminability contrasting co-located and mismatched intracranial locations of preceding sound and target. Significant differences were found for midline targets, for both binaural cues $(P=0.016$ for an ILD target, $P=0.011$ for an ITD target). For target locations to the left and right, discriminability did not differ significantly between preceding sound conditions (all $P>0.05$ ).

Individual results are displayed again in Figure 3, in a different format. This figure shows discriminability for ITD (upper panels) and ILD (bottom panels) as a function of the preceding sound condition. As suggested by the statistical analysis, results were averaged for both lateralized positions (left and right). A consistent pattern can be observed for midline targets: performance was better for a colocated preceding sound than for a mismatched preceding sound in five out of seven subjects for ITD (there was no effect in the remaining two subjects). For ILD, seven subjects out of seven demonstrated an advantage for co-located preceding sounds compared to mismatched preceding sounds. No such pattern was observed for lateral targets. In some listeners and conditions, the preliminary adjustment Step 3 failed to produce a $d^{\prime}$ of 2 for the condition without preceding sound in the main

\section{TABLE 2}

Mean and standard deviation for the just noticeable differences (JND) for subject, cue, and intracranial location (results of Step 2)

\begin{tabular}{|c|c|c|c|c|c|c|c|c|c|c|c|c|}
\hline \multicolumn{13}{|c|}{ Just noticable difference (JND) } \\
\hline \multirow{3}{*}{$\begin{array}{l}\text { Reference } \\
\text { Subject }\end{array}$} & \multicolumn{6}{|c|}{ ITD $[\mu s]$} & \multicolumn{6}{|c|}{$I L D[d B]$} \\
\hline & \multicolumn{2}{|c|}{ Left } & \multicolumn{2}{|c|}{ Middle } & \multicolumn{2}{|c|}{ Right } & \multicolumn{2}{|c|}{ Left } & \multicolumn{2}{|c|}{ Middle } & \multicolumn{2}{|c|}{ Right } \\
\hline & Mean & $S D$ & Mean & $S D$ & Mean & $S D$ & Mean & $\overline{S D}$ & $\overline{M e a n}$ & $\overline{S D}$ & Mean & $S D$ \\
\hline 1 & -69.02 & 21.60 & 40.57 & 5.70 & 91.97 & 30.55 & -4.48 & 1.32 & 5.48 & 2.08 & 5.09 & 0.86 \\
\hline 2 & -39.62 & 10.22 & 64.27 & 5.29 & 88.16 & 21.10 & -4.57 & 1.73 & 4.57 & 0.99 & 3.67 & 1.04 \\
\hline 3 & -61.55 & 17.04 & 62.84 & 17.15 & 112.44 & 37.12 & -3.58 & 0.44 & 5.84 & 2.03 & 1.92 & 0.79 \\
\hline 4 & -138.74 & 18.79 & 52.81 & 16.78 & 107.87 & 10.00 & -2.95 & 1.49 & 1.56 & 0.26 & 1.67 & 0.20 \\
\hline 5 & -38.73 & 8.34 & 29.27 & 7.51 & 44.81 & 15.04 & -2.98 & 0.80 & 2.12 & 0.54 & 3.34 & 0.87 \\
\hline 6 & -34.45 & 7.46 & 28.94 & 2.28 & 19.89 & 13.40 & -5.85 & 1.04 & 2.70 & 0.81 & 3.85 & 1.16 \\
\hline 7 & -125.82 & 38.82 & 83.60 & 23.13 & 114.05 & 42.72 & -5.89 & 1.57 & 5.27 & 1.56 & 6.72 & 1.27 \\
\hline
\end{tabular}


TABLE 3

Number of JNDs estimated to match discriminability without preceding sound for subject, binaural cue, and intracranial location (results of Step 3)

\begin{tabular}{|c|c|c|c|c|c|c|}
\hline \multicolumn{7}{|c|}{ Matched number of JNDs for $d^{\prime}=2$} \\
\hline \multirow[b]{2}{*}{ Reference } & \multicolumn{3}{|c|}{$I T D[\mu s]$} & \multicolumn{3}{|c|}{$I L D[d B]$} \\
\hline & Left & Middle & Right & Left & Middle & Right \\
\hline Subject & No. of JNDs & No. of JNDs & No. of JNDs & No. of JNDs & No. of JNDs & No. of JNDs \\
\hline 1 & 1.98 & 2.97 & 1.59 & 1.47 & 1.71 & 1.52 \\
\hline 2 & 2.45 & 1.64 & 0.58 & 0.75 & 0.74 & 1.27 \\
\hline 3 & 1.98 & 3.28 & 1.29 & 1.90 & 1.15 & 2.56 \\
\hline 4 & 1.84 & 3.25 & 2.04 & 1.77 & 8.54 & 2.76 \\
\hline 5 & 2.68 & 2.02 & 2.24 & 1.61 & 1.41 & 1.48 \\
\hline 6 & 2.35 & 2.50 & 4.36 & 0.06 & 2.20 & 1.39 \\
\hline 7 & 1.81 & 2.55 & 3.05 & 1.26 & 1.56 & 1.26 \\
\hline
\end{tabular}

experiment. These differences can often be traced back to particularly large or small differences in Table 4. However, there is no obvious change in the pattern of results depending on the accuracy of the adjustment step for a particular listener and condition, so this did not influence our conclusions.

Mean criterion (c) for each target location (left, middle, and right), binaural cue (ITD and ILD), and preceding sound condition (none, ITD left, ITD middle, and ITD right) is plotted in Figure 4. No obvious influence of the preceding sound could be observed on the criterion, which remains very close to zero (near optimal) in all cases. This was confirmed by three-way repeated-measures ANOVA with preceding sound condition, target location, and binaural cue as factors. The ANOVA did not reveal any significant shift of the criterion between preceding sound conditions $\left(P=0.423, F_{3,164}=0.982\right)$, between target locations $\left(P=0.212, F_{2,165}=1.772\right)$, or between the two binaural cues employed $(P=0.500$,
$\left.F_{1,166}=0.514\right)$. No significant interactions between any of the parameters employed were observed (all $P>0.05)$. In addition, we performed the same planned comparison as before to contrast colocated and mismatched conditions. The planned comparisons did not reveal any significant difference between co-located preceding sounds compared to preceding sounds located at a different intracranial position, for any binaural cue and target location. Thus, preceding sounds only influenced sensory sensitivity, and then only at midline, while the decision criterion was unaffected.

\section{DISCUSSION}

Investigating the effect of short preceding sounds on the discriminability of binaural cues in human listeners revealed three main findings. First, spatial discriminability can be improved when a sound precedes a

\section{TABLE 4}

Number of JNDs estimated to match discriminability without preceding sound for subject, binaural cue, and intracranial location (results of Step 3), expressed in terms of microseconds (ITD) and decibytes (ILD)

Change of binaural cue to be detected in the main experiment

\begin{tabular}{|c|c|c|c|c|c|c|}
\hline \multirow[b]{2}{*}{ Reference } & \multicolumn{3}{|c|}{ ITD [us] } & \multicolumn{3}{|c|}{$I L D[d B]$} \\
\hline & Left & Middle & $\overline{\text { Right }}$ & Left & Middle & $\overline{\text { Right }}$ \\
\hline Subject & $J N D$ & $J N D$ & JND & JND & $J N D$ & $J N D$ \\
\hline 1 & -136.66 & 120.49 & 146.23 & -6.59 & 9.37 & 7.74 \\
\hline 2 & -97.07 & 103.76 & 51.13 & -3.43 & 3.38 & 4.66 \\
\hline 3 & -121.87 & 206.12 & 145.05 & -6.80 & 6.72 & 4.92 \\
\hline 4 & -255.28 & 171.63 & 220.05 & -5.22 & 13.32 & 4.61 \\
\hline 5 & -103.80 & 59.13 & 100.37 & -4.80 & 2.99 & 4.94 \\
\hline 6 & -80.96 & 72.35 & 86.72 & -0.33 & 5.94 & 5.35 \\
\hline 7 & -125.82 & 213.18 & 347.85 & -7.42 & 8.22 & 1.60 \\
\hline
\end{tabular}


A

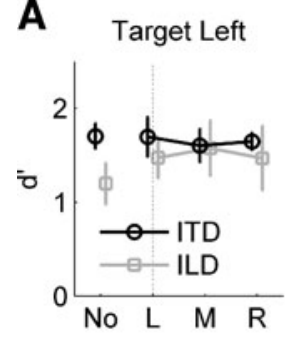

B

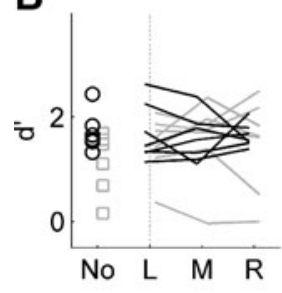

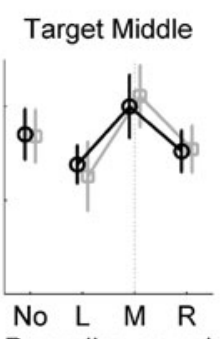

Preceding sound

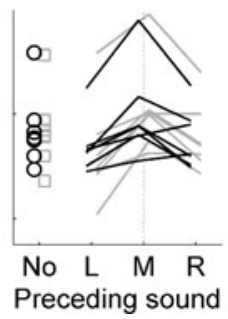

Target Right
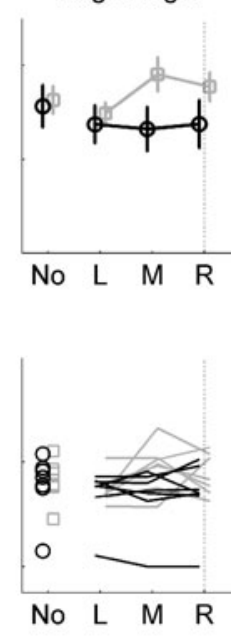

FIG. 2. A Mean discriminability and standard errors for each target location with respect to the presence/absence and intracranial location of the preceding sound. ITDs are plotted in black, ILDs in gray. B Same as A, but each line represents an individual listener.

target sequence at the same location compared to when they are mismatched in location. Second, this context effect only occurs for midline targets; no such effect is observed for targets located on the sides. Third, preceding sounds containing an ITD equally
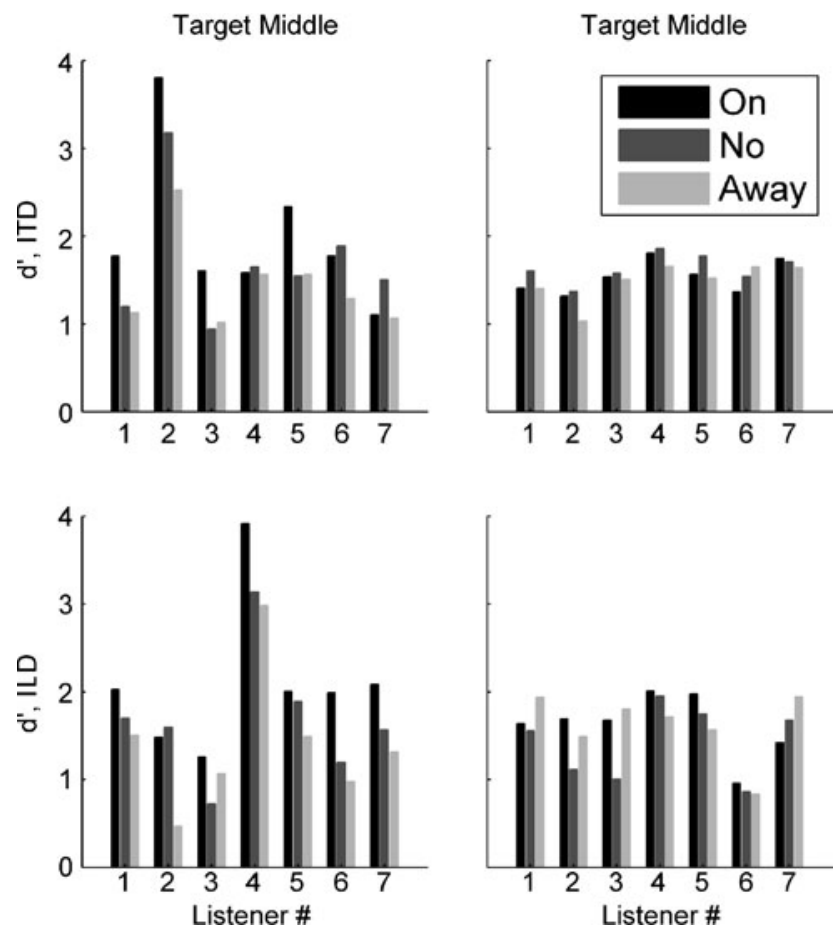

FIG. 3. Discriminability achieved by each subject for ITD (upper panels) and ILD (bottom panels) at middle (left panels) and lateral (right panels) target locations. Preceding sound conditions are depicted in black (co-located preceding sound and target), dark gray (no preceding sound), and light gray (mismatched preceding sound and target).

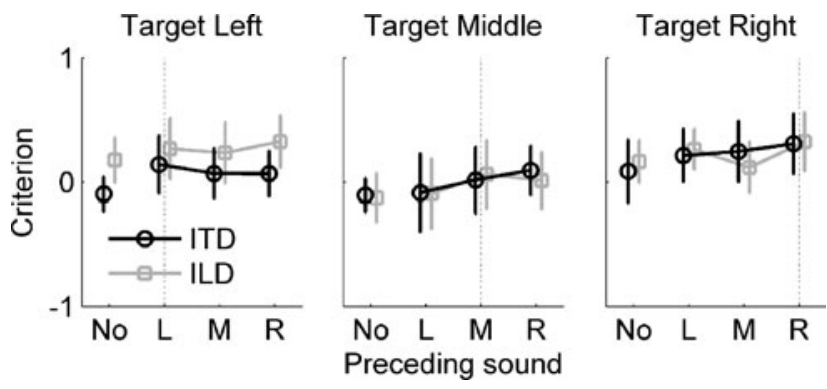

FIG. 4. Mean criterion and standard errors for each target location with respect to the location of the preceding sound. ITDs are plotted in black, ILDs in gray.

affect the discriminability of sounds lateralized by either ITD or ILD cues.

Preceding sounds influence spatial

discriminability only at midline

This is the first study of which we are aware that measures discriminability of intracranial location following exposure to a preceding ITD stimulus, using signal detection theory $\left(d^{\prime}\right)$ with a systematic crossing of binaural cues and subjective location. Our results show that midline discriminability of binaural cues improves significantly when the preceding sound and target sequence are co-located compared to when they are mismatched. The benefit relative to conditions without any preceding sound was not tested explicitly here to keep the number of planned comparisons feasible; moreover, if this benefit exists, the average data indicate that it is relatively small. However, realistic listening situations usually involve a continuous stream of sounds, so the comparison between mismatched and co-located conditions is arguably the more relevant one. This comparison results in a sizeable average gain in discrimination performance, ranging from a $30 \%$ to a $70 \%$ increase in $d^{\prime}$, depending on the condition. In general, the improvements we observe in discrimination performance following a preceding sound are consistent with previous findings. Kashino (1998) reported an improvement of ITD thresholds after presentation of an ITD stimulus of $0 \mu \mathrm{s}$. Other studies, using different experimental methods, reported improvements in minimum audible angle (Getzmann 2004) or discriminability (Sach et al. 2000).

In contrast to the improvements found by Getzmann (2004) for lateralized targets or with the cueing paradigms that used a lateralized cue (Sach et al. 2000), we did not find that lateralized targets were influenced by a preceding sound. Does this difference derive from a lack of statistical power of our analyses? For instance, there seems to be a nonsignificant trend for elevated discriminability for right-lateralized tar- 
gets preceded by sounds containing an ITD located at midline or to the right (Fig. 2A, right panel). However, the same analysis method showed a clear effect on midline targets, so if the effect exists for lateral targets, it must be much smaller or less reliable. The individual data of Figures 2B and 3 strongly suggest that for the great majority of listeners the impact of the preceding sound was indeed less effective in influencing discrimination for lateralized targets than for middle targets.

There is an important difference between this and previous studies that could explain the lack of effect for lateralized targets in our study. Sach et al. (2000) only observed an effect for lateral targets when topdown expectations were recruited, by blocking trials based on the location of the preceding sound (Experiments 2-5). Getzmann (2004) also only used a single preceding sound location, which amounts to blocking. Our results suggest that when the position of the preceding sound changes randomly within a block so that it is not indicative of the target position, discriminability changes at midline, but no influence is observed for lateralized targets.

That the effect of context is only observed around the midline may be consistent with the importance of midline positions for sound localization. Auditory spatial resolution is most accurate around the midline (e.g., Mills 1958), so a useful strategy under free-field listening conditions would be to orient the head toward the general location of any sound of interest. In natural conditions, it may, therefore, be the case that any important localization task that begins with lateral targets is often rendered frontal because of head movements.

Possible neural correlates for the effect of preceding sounds lateralized by ITDs on subsequent ITD targets

What brain mechanisms might contribute to differences in discriminability of ITD cues in a manner dependent on the ITD of a preceding stimulus? A possible candidate is the adaptive mechanism recently reported for the encoding of ITD cues in electrophysiological investigations. Maier et al. (2007) reported that neural coding accuracy for ITDs could change as a function of the prevailing context. They recorded responses to inferior colliculus neurons to ITDs that changed dynamically every $50 \mathrm{~ms}$, with a high-probability region (HPR) around a restricted range of ITD values. An analysis of Fisher Information (FI), which measures coding accuracy, taking into account how local changes in the stimulus affect both mean and variance of neural firing rate (Dayan and Abbott 2001) was performed on the neural responses. High FI at one stimulus value predicts high neural discriminability around this value. Maier et al. (2007) found that highest coding accuracy was observed for midline ITDs. Furthermore, the changes in the location of the HPR induced changes in the maximum of the FI towards the HPR. Finally, and importantly, when both brain hemispheres were considered (i.e., the results from recordings in the right IC were mirrored to reflect the presumed response of the left IC) changes in FI because of adaptive coding were only observed for positions around the midline.

The adaptive effect observed in the physiology is thus compatible with many features of the behavioral data in this study. The preceding context only affected discrimination performance for locations near or at midline. The electrophysiological paradigm operated on time scales similar to the psychophysical procedure reported here, even though there was no temporal gap between the preceding sound and test stimulus. Our perceptual data are thus consistent with a purely bottom-up change in the encoding of ITD localization cues that operates on a relatively short time scale and that primarily affects midline positions.

The parallel between psychophysical data and adaptive coding is suggestive, but the fact that the two datasets were observed in different species (human vs. guinea pig) and vigilance state (awake vs. anaesthetized) must qualify the conclusions. Moreover, in the behavioral data reported here, response strategies might have influenced the pattern of results. It is possible that listeners were able to use absolute location cues for targets at midline. Specifically, a preceding sound at midline could provide a top-down perceptual anchor for an absolute scale, especially as there was no location rove. Such a strategy might not be available for lateralized targets as their absolute location is usually less precisely defined. Such a strategy also is consistent with the fact that the effect was not cue-specific. The neural mechanisms subtending these anchoring effects, although unspecified, are likely to be substantially different from the purely bottom-up ones described above. Although this alternative interpretation cannot be ruled out, we think it unlikely for the following reasons. In our experiment, trials were randomly interleaved with respect to the target and preceding sound positions. A putative anchoring of the "midline scale" would thus have to have been maintained across several trials where the midline position was not presented. In addition, if an anchoring mechanism had been active, absolute position would also have been encoded (at least to some extent) for sounds on the side. However, lateral preceding sounds did not lead to an improvement in performance for lateral targets. Finally, absolute localization is known to be worse than relative localization, which 
renders the usage of an absolute scale an inefficient strategy to perform the task.

Yet, another response strategy can be entertained. It is possible that listeners focused their attention on the location of the preceding sound, thus improving performance at the focused location and impairing performance at all other locations. Such covert shifts of attention have been shown to speed reaction times when the location of a task is cued by a preceding sound (Spence and Driver 1994). However, in our experiment, the effects of preceding sounds were only observed when the target was at midline. Thus, if this explanation is correct, covert shifts of attention must only be produced when the target is at midline. But, the preceding sound was not informative of the position of the targets, so listeners could not have predicted which trials were going to be midline trials. The failure of covert shifts of attention to affect performance for lateral targets cannot be attributed to a floor effect either, as discriminability was equated for all positions.

In summary, our results can be parsimoniously explained by a purely bottom-up neural mechanism that is only effective around the midline. Other mechanisms, some of them top-down, may be recruited in listening conditions where preceding sounds might have an informative content about the task to be performed (Spence and Driver 1994; Sach et al. 2000) but are unlikely to have contributed to the current results.

\section{Interaction between ITD and ILD cues}

In addition to influencing the lateralization of ITD targets, preceding sounds containing an ITD were also found to influence discriminability of noise bursts lateralized by means of ILDs. The similar influence of the ITD preceding sound on both binaural cues is consistent with the notion that neural processing of ITDs and ILDs interacts somewhere in the auditory brain (e.g., Hari 1995; Phillips et al. 2006) such that an integrated representation of auditory space emerges, rather than a representation of the binaural cues per se. Our behavioral results imply that the neural basis of the effect of a preceding sound is located at or after the level at which the two binaural cues converge.

One possibility for how this convergence could be effected is time-intensity trading, where differences in level produced by ILDs effect changes in neural latency, resulting in ITDs in the internal representation. Time-intensity trading has been observed at the level of the auditory nerve (e.g., Heil 2004; Joris et al. 2008), the auditory brainstem (Joris and Yin 1995; Tollin and Yin 2005; Yin and Chan 1990; for reviews, see e.g., Grothe 2003; Yin 2002), and the inferior colliculus in the midbrain (Irvine et al. 1995). Although these findings already indicate some blurring of the distinction between ITD and ILD within the early pathways, no clear pattern of trading ratios could be found at any stage (Irvine et al. 1995; Joris and Yin 1995; Joris et al. 2008). Moreover, the extent of the trading observed physiologically is insufficient to explain the perceptual influence of ILDs. It, thus, seems unlikely that our results can be explained by time-intensity trading alone.

Convergence of the representation of binaural cues could also occur in the IC itself, as it receives projections from both the medial and lateral superior olive, where ITD and ILD are thought to be first represented (Kuwada and Yin 1983; Yin and Kuwada 1983). Further, candidates are the medial geniculate body (e.g., Jones et al. 2007) and fields in the auditory cortex (e.g., Jenkins and Merzenich 1984; Stecker et al. 2003; Stecker and Middlebrooks 2003; Deouell 2007). In particular, the secondary auditory cortex contains areas in which sound localization cues may be merged (Stecker et al. 2005).

The finding that an ITD context affects ITD and ILD cues equally is an important constraint on the neural bases of the behavioral context effect. However, given the current lack of consensus as to where binaural cues first converge in the auditory pathways, and on the potential effects of feedback on lower stations, the current results do not provide strong constraints on the most likely neural site for spatial context coding.

\section{ACKNOWLEDGEMENTS}

We thank Clara Suied for helpful comments on a previous version of the manuscript. We are also grateful to Barbara Shinn-Cunningham, acting as Associate Editor, for her helpful comments. This study was supported by a DFG grant (SFB/TRR31), the MRC, and grant ANR-06-Neuro-022-01.

\section{REFERENCES}

Carlile S, Hyams S, Delaney S (2000) Systematic distortions of auditory space perception following prolonged exposure to broadband noise. J Acoust Soc Am 110:416-424

Dayan P, Аввотт LF (2001) Theoretical neuroscience: computational and mathematical modeling of neural systems. MIT, Cambridge

Dean I, Harper NS, McAlpine D (2005) Neural population coding of sound level adapts to stimulus statistics. Nat Neurosci 8:16841689

Dean I, Robinson BL, Harper NS, McAlpine D (2008) Rapid neural adaptation to sound level statistics. J Neurosci 28:6430-6438

Deouell LY (2007) Cerebral responses to change in spatial location of unattended sounds. Neuron 55:985-996

Dong C-J, Swindale NV, Cynader MS (1999) A contingent aftereffect in the auditory system. Nat Neurosci 2:863-865 
Fairhall AL, Lewen GD, Bialek W, de Ruyter van Steveninck RR (2001) Efficiency and ambiguity in an adaptive neural code. Nature 412:787-792

FurukaWa S, Maki K, Kashino M, Riquimaroux H (2005) Dependency of the interaural phase difference sensitivities of inferior collicular neurons on a preceding tone and its implications in neural population coding. J Neurophysiol 93:3313-3326

Getzmann S (2004) Spatial discrimination of sound sources in the horizontal plane following an adapter sound. Hear Res 191:14-20

Green DM, Swets JA (1966) Signal detection theory and psychophysics. Wiley, Oxford

Grothe B (2003) New roles for synaptic inhibition in sound localization. Nat Rev Neurosci 4:540-550

HARI R (1995) Illusory directional hearing in humans. Neurosci Lett 189:29-30

HeIL P (2004) First-spike latency of auditory neurons revisited. Curr Opin Neurobiol 14:461-467

Irvine DRF, Park VN, Mattingley JB (1995) Responses of neurons in the inferior colliculus of the rat to interaural time and intensity differences in transient stimuli. Implications for the latency hypothesis. Hear Res 85:127-141

Jenkins WM, MERZENICH MM (1984) Role of cat primary auditory cortex for sound-localization behavior. J Neurophysiol 52:819-847

Jones S, Nakamoto K, Palmer A (2007) Contrasting effects of cortical inactivation on medial geniculate body and inferior colliculus. Abstr 30th Midwinter Meeting Assoc Res Otolaryngol 692:238

JoRIS PX, YIN TCT (1995) Envelope coding in the lateral superior olive. I. Sensitivity to interaural time differences. J Neurophysiol 73:1043-1062

Joris PX, Michelet P, Franken TP, McLaughuin M (2008) Variations on a dexterous theme: peripheral time-intensity trading. Hear Res 238:49-57

KASHINO M (1998) Adaptation in sound localization revealed by auditory after-effects. In: Palmer AR, Rees A, Summerfield AQ, Meddis R (eds) Psychophysical and physiological advances in hearing. Whurr, London, pp 322-328

Kashino M, Nishida S (1998) Adaptation in the processing of interaural time differences revealed by the auditory localization aftereffect. J Acoust Soc Am 103:3597-3604

KuwADA S, YIN TCT (1983) Binaural interaction in low-frequency neurons in inferior colliculus of the cat. I. Effects of long interaural delays, intensity, and repetition rate on interaural delay function. J Neurophysiol 50:981-999

Macmillan NA, Creelman CD (2005) Detection theory. A user's guide, 2nd edn. Erlbaum, Mahwah

Maier JK, Harper NS, Dean I, Klump G, McAlpine D (2007) Adaptive coding of interaural time differences in the auditory midbrain. Abstr 30th Midwinter Meeting Assoc Res Otolaryngol 418:145
Malone BJ, Scott BH, Semple MN (2002) Context-dependent adaptive coding of interaural phase disparity in the auditory cortex of awake maqaques. J Neurosci 22:4625-4638

Mills AW (1958) On the minimum audible angle. J Acoust Soc Am 30:237-246

Mossop JE, Culling JF (1998) Lateralization of large interaural delays. J Acoust Soc Am 104:1574-1579

Phillips DP, Hall SE (2005) Psychophysical evidence for adaptation of central auditory processors for interaural differences in time and level. Hear Res 202:188-199

Phillips DP, Carmichael ME, Hall SE (2006) Interaction in the perceptual processing of interaural time and level differences. Hear Res 211:96-102

SACH AJ, Hill NI, Bailey PJ (2000) Auditory spatial attention using interaural time differences. J Exp Psychol 26:717-729

Spence CJ, Driver J (1994) Covert spatial orienting in audition: exogenous and endogenous mechanisms. J Exp Psychol 20:555574

Stecker GC, Middlebrooks JC (2003) Distribute coding of sound locations in the auditory cortex. Biol Cybern 89:341-349

Stecker GC, Mickey BJ, Macpherson EA, Middlebrooks JC (2003) Spatial sensitivity in field PAF of cat auditory cortex. J Neurophysiol 89:2889-2903

Stecker GC, Harrington IA, Middlebrooks JC (2005) Location coding by opponent neural populations in the auditory cortex. Plos Biol 3:520-528

TERHardt E (1968) Über ein Äquivalenzgesetz für Intervalle akustischer Empfindungsgrössen. Kybernetik 5:127-133

Thurlow WR, JACK CE (1973) Some determinants of localizationadaptation effects for successive auditory stimuli. J Acoust Soc Am 53:1573-1577

TolLin DJ, YIN TCT (2005) Interaural phase and level difference sensitivity in low-frequency neurons in the lateral superior olive. J Neurosci 25:10648-10657

Ulanovsky N, Las L, Nelken I (2003) Processing of low-probability sounds by cortical neurons. Nat Neurosci 6:330-332

Vigneault-Maclean BK, Hall SE, Phillips DP (2007) The effects of lateralized adaptors on lateral position judgments of tones within and across frequency channels. Hear Res 224:93-100

YIN TCT (2002) Neural mechanisms of encoding binaural localization cues in the auditory brainstem. In: Oertel D, Fay RR, Popper AN (eds) Integrative functions in the mammalian auditory pathway. Springer, New York, pp 99-159

YIN TCT, CHAN JCK (1990) Interaural time sensitivity in medial superior olive of cat. J Neurophysiol 64:465-488

YIN TCT, KuwADA S (1983) Binaural interaction in low-frequency neurons in inferior colliculus of the cat. II. Effects of changing rate and direction of interaural phase. J Neurophysiol 50:1000-1019 